\title{
Transmembrane Potential of Physiologically Relevant Model Membranes: Effects of Membrane Asymmetry
}

Xubo Lin ${ }^{1, *}$ and Alemayehu A. Gorfe $\mathrm{e}^{2, *}$

1. Institute of Single Cell Engineering, Beijing Advanced Innovation Center for Biomedical Engineering, Beihang University, Beijing 100191, China

2. Department of Integrative Biology and Pharmacology, McGovern Medical School, The University of Texas Health Science Center at Houston, Texas 77030, USA

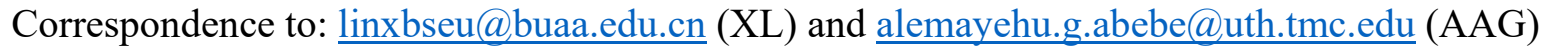

The authors declare no competing financial interest.

\section{TOC Graphics}

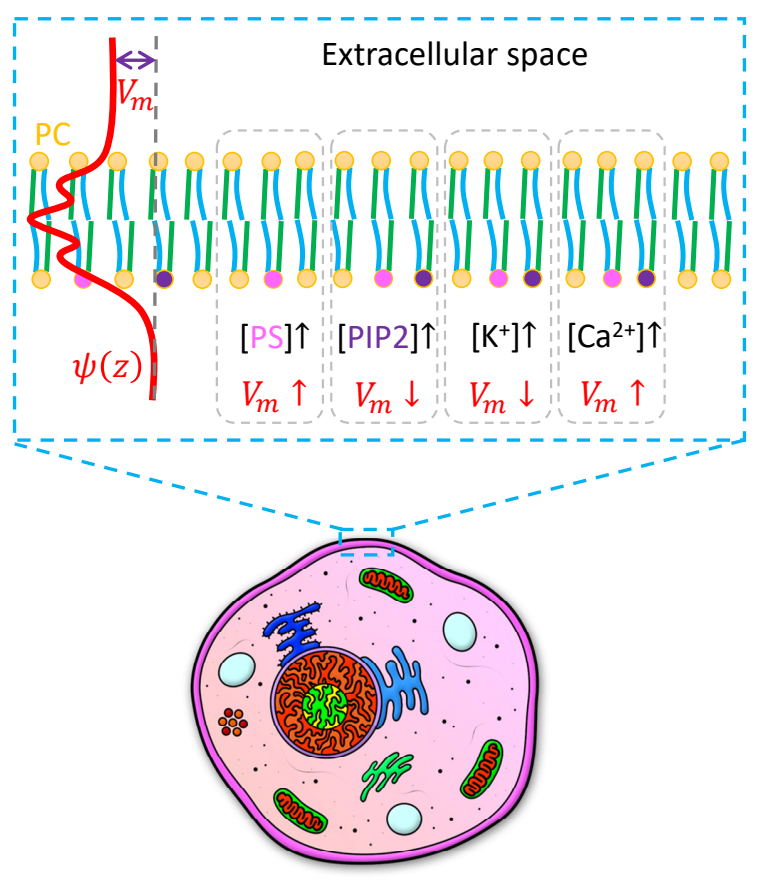

Transmembrane potential is modulated by membrane asymmetry. 


\begin{abstract}
Transmembrane potential difference $\left(V_{m}\right)$ plays important roles in regulating various biological processes. At the macro level, $V_{m}$ can be experimentally measured or calculated using the Nernst or Goldman-Hodgkin-Katz equation. However, the atomic details responsible for its generation and impact on protein and lipid dynamics still need to be further elucidated. In this work, we performed a series of all-atom molecular dynamics simulations of symmetric model membranes of various lipid compositions and cation contents to evaluate the relationship between membrane asymmetry and $V_{m}$. Specifically, we studied the impact of the asymmetric distribution of POPS (1palmitoyl-2-oleoyl-sn-glycero-3-phospho-L-serine), PIP2 (phosphatidylinositol 4,5-bisphosphate), $\mathrm{Na}^{+}, \mathrm{K}^{+}$and $\mathrm{Ca}^{2+}$ on $V_{m}$ using atomically detailed molecular dynamics simulations of symmetric model membranes. The results suggest that, for an asymmetric POPC-POPC/POPS bilayer in the presence of $\mathrm{NaCl}$, enrichment of the monovalent anionic lipid POPS in the inner leaflet polarizes the membrane $\left(\Delta V_{m}<0\right)$. Intriguingly, replacing a third of the POPS lipids by the polyvalent anionic signaling lipid PIP2 counteracts this effect, resulting in a smaller negative membrane potential. We also found that replacing $\mathrm{Na}^{+}$ions in the inner region by $\mathrm{K}^{+}$depolarizes the membrane $\left(\Delta V_{m}>0\right)$, whereas replacing by $\mathrm{Ca}^{2+}$ polarizes the membrane. These divergent effects arise from variations in the strength of cation-lipid interactions and are correlated with changes in lipid chain order and head group orientation.
\end{abstract}

Keywords: Transmembrane Potential; Membrane Asymmetry; Lipid Composition; Ion Distribution

\title{
Introduction
}

The physiologic transmembrane potential $\left(V_{m}\right)$ represents the difference in electrical potential between the extracellular and intracellular compartments of the cell. The magnitude of the $V_{m}$ in resting cells varies from cell to cell but generally ranges from about $-10 \mathrm{mV}$ to $-100 \mathrm{~m} \mathrm{~V}^{[1]}$. It is well known that fluctuations in electrical signal play many essential roles in the cell. For instance, in nerve cells, changes in $V_{m}$ regulate the amplitude and duration of somatic and axonal action potentials $^{[2]}$. Changes in $V_{m}$ can also alter the membrane permeability of many ions and other small molecules ${ }^{[3]}$ that are critical for cell fate decisions and maintenance of the homeostatic balance $\mathrm{e}^{[4-5]}$. Therefore, cells employ sophisticated mechanisms, such as the regulated opening and closing of ion channels, to control the extent of plasma membrane depolarization (reduction of $V_{m}$ ) or hyperpolarization (larger negative $V_{m}$ ). A phenomenon less well-understood at the molecular level is how changes in $V_{m}$ might alter the organization of plasma membrane lipids and proteins to modulate signaling events underlying cell growth and proliferation. This fundamental biophysical problem has important implications to pathophysiology including cancer. For example, it has been shown that depolarization enhances the nanoscale clustering and activation of the oncogenic protein K-Ras, resulting in enhanced signaling and cell proliferation ${ }^{[6]}$. This was proposed to arise 
from changes in the lateral dynamics of 1-palmitoyl-2-oleoyl-sn-glycero-3-phospho-L-serine (POPS) and phosphatidylinositol 4,5-bisphosphate (PIP2) lipids ${ }^{[6]}$. Therefore, it is important to understand not only the atomic basis of $V_{m}$ but also how it might depend on and, in turn, affect the structure and dynamics of membrane components. The focus of the current work is to examine the effect of membrane compositional asymmetry and ion distribution on $V_{m}$.

Although its exact lipid composition may depend on cell status and varies from cell type to cell type, a large body of previous research has established a general picture of the plasma membrane lipid composition ${ }^{[7-8]}$ : (1) some of the most common phospholipids such as 1-palmitoyl-2-oleoylsn-glycero-3-phosphocholine (POPC) are found on both sides of the plasma membrane, (2) the intracellular leaflet is enriched in anionic lipids such as POPS, and (3) there are more sodium ions in the extracellular space and more potassium ions in the cytosol. Studies have also shown that $\mathrm{Ca}^{2+}$ affects the head-group conformation ${ }^{[9]}$ as well as the dynamics and clustering ${ }^{[10]}$ of PIP2, a signaling lipid involved in a wide variety of biological processes ${ }^{[1]}$. Moreover, intracellular $\mathrm{Ca}^{2+}$ regulates the structure and dynamics of many membrane proteins including those involved in transport of ions across the plasma membrane ${ }^{[12-13]}$. We therefore systematically studied the impact of the asymmetric distribution of POPS, PIP2, $\mathrm{Na}^{+}, \mathrm{K}^{+}$and $\mathrm{Ca}^{2+}$ on $V_{m}$ using atomically detailed molecular dynamics (MD) simulations. The results suggest that, for an asymmetric PC-PC/PS bilayer in the presence of $\mathrm{NaCl}$ salt, the accumulation of POPS in the inner leaflet polarizes the membrane $\left(\Delta V_{m}<0\right)$. Interestingly, replacing a third of the PS lipids by PIP 2 counteracts this effect, resulting in a smaller negative membrane potential. Intracellular $K^{+}$depolarizes the membrane $\left(\Delta V_{m}>0\right)$ while intracellular $\mathrm{Ca}^{2+}$ dramatically polarizes the membrane. These effects arise from differences in the strength of cation-lipid interactions, and are correlated with changes in lipid chain order and head group orientation.

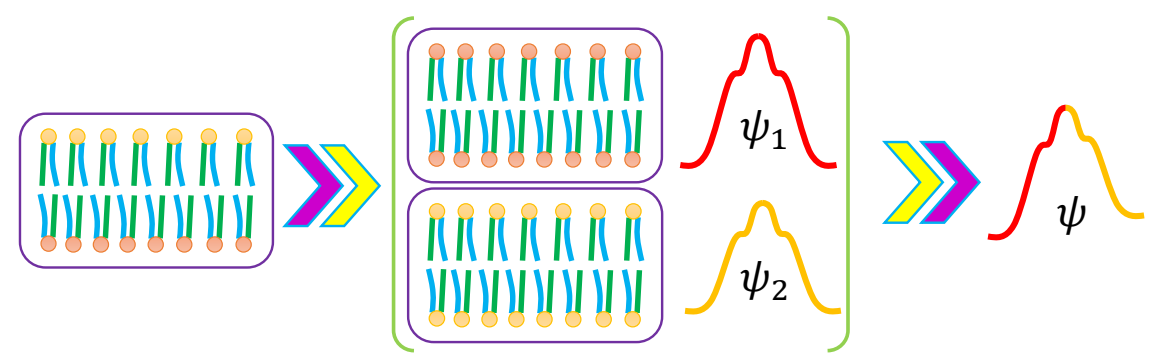

Scheme 1. Estimating the electrostatic potential $(\psi)$ of an asymmetric membrane using two symmetric membranes.

\section{Methods}

MD simulation is a powerful tool to probe the atomic details associated with transmembrane potential $^{[14-15]}$ generation and impact. Previous MD simulations used coarse-grained ${ }^{[16]}$, unitedatom $^{[17]}$, all-atom ${ }^{[18]}$ and polarizable ${ }^{[19]}$ models to estimate the $V_{m}$ of a variety of lipid bilayers. These models yielded dramatically different electrostatic potential profiles, but the final $V_{m}$ values were generally comparable to experiments. Here, we chose to use an all-atom model ${ }^{[20]}$ in order to 
capture the motion of every atom in a comparatively cost-effective manner (relative to polarizable force fields).

Calculating $V_{m}$ of an Asymmetric Membrane from Simulations of Two Symmetric Membranes. An accurate calculation of $V_{m}$ from MD simulation depends on how well the charge distribution is sampled along the membrane normal. However, it is not always easy to achieve convergence of the charge distribution. Let us consider, for example, a two-bilayer all-atom simulation system with no charge imbalance between leaflets $(\Delta Q=0)$. If this system is made up of single-component symmetric lipids, it is relatively easy to achieve convergence within a simulation time of hundreds of nanoseconds. This would yield a high-quality electrostatic potential profile $^{[14,17]}$ that is mirror-symmetric with respect to the mid-plane of the bilayer, and the final $V_{m}$ would be zero, as expected. However, if the bilayer is composed of multiple lipid types, which is more physiological, even a $\mu s$-scale simulation is not sufficient to obtain a mirror-symmetric electrostatic potential profile. For example, one study found an unacceptably high $V_{m} \approx 40 \mathrm{mV}$ with $\Delta Q=0$ (excepted: $\left.V_{m} \approx 0 \mathrm{mV}\right)^{[18]}$. In asymmetric bilayers, the problem is compounded by several factors. (i) Lipid compositional asymmetry can result in local bilayer deformation, making the accurate calculation of charge density along the membrane normal difficult. (ii) Ions can passively equilibrate across the membrane, making it difficult to maintain a differential distribution. To avoid these complications and obtain a reliable estimate of $V_{m}$ for multicomponent asymmetric membranes, two separate simulations can be conducted: one representing the outer leaflet of the plasma membrane and another representing the inner leaflet. Then, in each case, the charge density distribution $\rho(z)$ ( $\mathrm{z}=0$ being the bilayer center) is averaged over the two halves (effectively symmetrized with respect to the membrane center of mass) as $\rho(z)=(\rho(-z)+\rho(+z)) / 2$. Thus, for the individual symmetric systems, the symmetrized $\rho(z)$ results in symmetric $\psi(z)$ and thus eliminates a non-zero $V_{m}$. To model the $V_{m}$ of asymmetric membranes, we combined the symmetric $\psi(z)$ profiles of two symmetric membrane systems (Scheme 1) such that one represents the region $\mathrm{z}<0$ and the other $\mathrm{z} \geqslant 0$; the recombinant $\psi(z)$ then yields an effective transmembrane potential for the asymmetric membrane ${ }^{[21]}$. We used this approach to estimate the electrostatic potential of four asymmetric membrane systems based on the bilayers listed in Table 1.

MD Simulation. The CHARMM36 force field ${ }^{[20]}$ was used to perform all-atom MD simulations on five symmetric single-bilayer systems (Table 1). The initial configuration for each system was prepared using CHARMM-GUI ${ }^{[22-23]}$. The simulations were run under the constant pressure and temperature (NPT) ensemble with lipids, water and ions separately coupled to a Nose-Hoover heat bath $^{[24-25]}$ at $\mathrm{T}=310 \mathrm{~K}$ (coupling constant $\tau=1 \mathrm{ps}$ ) and a pressure of 1 bar maintained by a semiisotropic Parrinello-Rahman pressure coupling scheme ${ }^{[26]}$ (coupling constant $\tau=5$ ps and compressibility $\left.=4.5 \times 10^{-5} \mathrm{bar}^{-1}\right)$. The leap-frog Verlet algorithm, and a periodic boundary condition (with bonds involving hydrogen atoms constrained using the LINCS algorithm ${ }^{[27]}$ ) were used. The Lennard-Jones potential was smoothly shifted to zero between 1.0 and $1.2 \mathrm{~nm}$, and particle mesh Ewald (PME) electrostatics ${ }^{[28]}$ was used with a real space cutoff of $1.2 \mathrm{~nm}$. The nonbonded interaction neighbor list was updated every 20 steps with a cutoff of $1.2 \mathrm{~nm}$. Simulations were run using GROMACS (version 2016.04) ${ }^{[29]}$ for $350 \mathrm{~ns}$ with a time step of $2 \mathrm{fs}$, and coordinates were saved every 4 ps for analysis. Snapshots were rendered using $\mathrm{VMD}^{[30]}$. 
Table 1. Symmetric Membrane Systems Simulated in This Work.

\begin{tabular}{|c|c|c|c|c|c|c|c|}
\hline \multirow{2}{*}{ System Name } & \multicolumn{3}{|c|}{ No. of Lipids } & \multicolumn{5}{c|}{ No. of ions } \\
\cline { 2 - 9 } & POPC & POPS & PIP2* & $\mathrm{Na}^{+}$ & $K^{+}$ & $\mathrm{Ca}^{2+}$ & $\mathrm{Cl}^{-}$ \\
\hline PC & 200 & 0 & 0 & 32 & 0 & 0 & 32 \\
\hline PC/PS & 140 & 60 & 0 & 92 & 0 & 0 & 32 \\
\hline PC/PS/PIP2 & 140 & 40 & 20 & 172 & 0 & 0 & 32 \\
\hline PC/PS/PIP2/K & 140 & 40 & 20 & 0 & 172 & 0 & 32 \\
\hline PC/PS/PIP2/Ca & 140 & 40 & 20 & 0 & 0 & 86 & 32 \\
\hline
\end{tabular}

* Data for protonated PIP2 is shown in SI. Each simulation was run for $350 \mathrm{ns,}$ and the first 50 ns was considered equilibrium phase while the last $300 \mathrm{~ns}$ data was used for analysis by dividing into six $50 \mathrm{~ns}$ blocks. Each system was charge-neutralized and the $\mathrm{Cl}^{-}$concentration is $150 \mathrm{mM}$.

\section{Trajectory Analysis.}

Mass/Charge Density Calculation. Electrostatic potential $\psi(z)$ is calculated as the double integral of the charge density $\rho(z)$ along the membrane normal $(z)$ using the Poisson equation:

$$
\psi(z)=-\frac{1}{\varepsilon_{0}} \int_{-z}^{z} d z^{\prime} \int_{-z^{\prime}}^{z^{\prime}} \rho\left(z^{\prime \prime}\right) d z^{\prime \prime}
$$

The accuracy of $\psi(z)$ depends on the calculation of $\rho(z)$. In our NPT simulations, the z-dimension of the simulation box fluctuates and the z-location of the membrane center of mass may change over time. Hence, a direct application of the GROMACS tool gmx_mpi density, which bins the membrane along the z-dimension of the simulation box to calculate the mass and charge density profiles, may give rise to an inaccurate $\rho(z)$. Instead, we used an in-house script (based on " $x d r f i l e-$ 1.1.4", an open source program) that re-calculates the membrane center $(z=0 \mathrm{~nm})$ and equally divides the range $|\Delta z|<4 \mathrm{~nm}$ to obtain the mass or charge distribution for each trajectory frame (bin width of $0.05 \mathrm{~nm}$ was used in the current work). The final mass or charge density profile is obtained by averaging over all frames.

Lipid Acyl Chain Order Parameter. As a direct measure of the structural flexibility of lipids, the lipid chain order parameter, $S_{C H}$, can be calculated as follows:

$$
S_{C H}=\frac{1}{2}\left\langle 3 \cos ^{2} \theta-1\right\rangle
$$

Lipid Head Tilt Angle. For POPC and POPS lipids, the vector connecting the $\mathrm{P}$ atom and $\mathrm{N}$ atom was used to quantify lipid head orientation. For PIP2, the vector connecting the first and fourth carbon atoms in the six-membered carbon ring was used. The angle between these vectors and the membrane normal was defined as lipid head tilt angle, which was calculated using an in-house script. 
Lipid-Ion Contact Probability. Contacts between atoms in lipids and ions were counted when the distance between the was less than $0.6 \mathrm{~nm}$ and contact probability was then obtained by averaging over lipids and frames of the last $300 \mathrm{~ns}$ trajectories. The final snapshot visualization was achieved by $\mathrm{VMD}^{[30]}$ using "Beta" coloring method, where the "B-factor" columns in the $p d b$ files of lipids were replaced with the calculated contact probability.
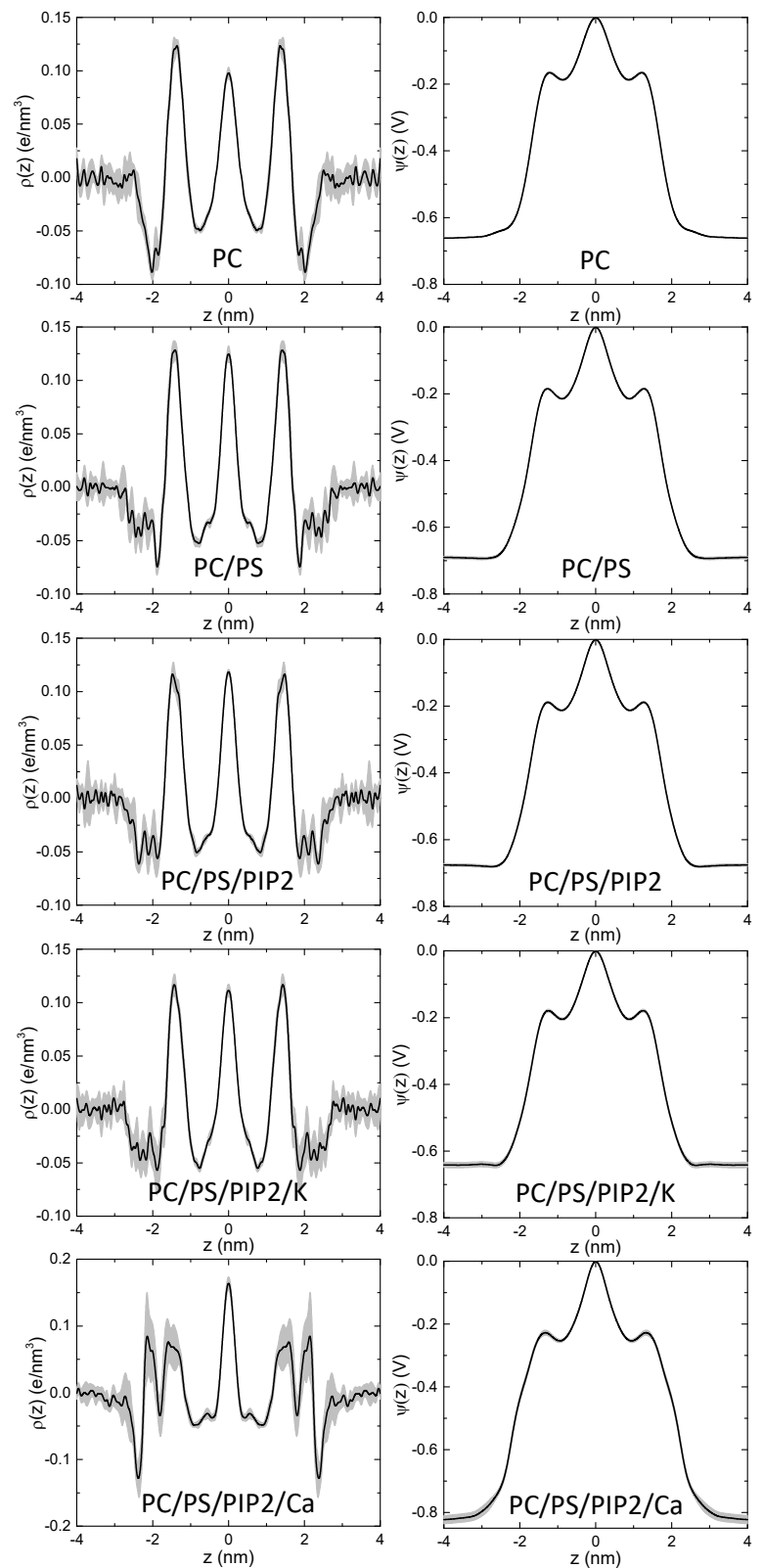

Figure 1. Charge density distribution (left column) and electrostatic potential (right column) profiles of the five symmetric systems in this work. Bin width of $0.05 \mathrm{~nm}$ is used, and the error bars are shown in gray shade.

\section{Results and Discussion}


$V_{m}$ of Asymmetric Membranes from Symmetric Model Membranes. Previous MD studies have shown that it is difficult to obtain a symmetric electrostatic potential profile for multi-component bilayers. This is likely due to insufficient sampling ${ }^{[18]}$. Modeling the $V_{m}$ of an asymmetric bilayer presents additional technical challenges. Therefore, we followed a similar approach to that used by Falkovich et al. ${ }^{[21]}$ and simulated symmetric membranes whose lipid composition roughly models the inner and outer leaflets of the plasma membrane to estimate the $V_{m}$ of asymmetric model membranes (see Methods). We used a pure POPC bilayer as a simplified model of the plasma membrane outer leaflet. The inner leaflet is modeled by a two-component lipid bilayer POPC/POPS (0.7:0.3) or a three-component bilayer POPC/POPS/PIP2 (0.7:0.2:0.1), reflecting the enrichment of these anionic lipids in the inner leaflet of the plasma membrane. These three systems were simulated in the presence of $\mathrm{NaCl}$, and are referred to as PC, PC/PS and PC/PS/PIP2 (Table 1). To examine the impact of the higher concentration of potassium and calcium ions in the inner leaflet of the plasma membrane, we ran two additional simulations in which $\mathrm{Na}^{+}$was replaced by $\mathrm{K}^{+}$or $\mathrm{Ca}^{2+}$ so that the total number of positive charges remains constant (systems PC/PS/PIP/K and $\mathrm{PC} / \mathrm{PS} / \mathrm{PIP} / \mathrm{Ca}$ ).

Fig. 1 (left panel) shows the charge density distribution along the membrane normal, $\rho(z)$, for each of the five systems simulated in this work. Note that for each frame of the last $300 \mathrm{~ns}$ of each trajectory, $\rho(z)$ was symmetrized over the two halves of the bilayer centered at $z=0$. Using equation 1 and setting $\psi(0)=0 \mathrm{mV}$, we obtained the corresponding symmetric electrostatic potential $(\psi(z))$ profiles Fig. 1 (right panel). As illustrated in Scheme 1, each $\psi(z)$ profile derived from each model membrane representing the inner leaflet of the plasma membrane (systems PC/PS, $\mathrm{PC} / \mathrm{PS} / \mathrm{PIP} 2, \mathrm{PC} / \mathrm{PS} / \mathrm{PIP} / \mathrm{K}$ and PC/PS/PIP/Ca) was combined with that of the outer leaflet $\psi(z)$ (system PC), to obtain recombinant $\psi(z)$ profiles for the asymmetric PC-PC/PS, PC-PC/PS/PIP2, PC-PC/PS/PIP2/K and PC-PC/PS/PIP2/Ca membranes (Fig. 2a). In these plots, $-4<\mathrm{z}<0 \mathrm{~nm}$ represents the outer leaflet of the membrane made up of pure PC lipids while the region $0<\mathrm{z}<4$ $\mathrm{nm}$ presents the inner leaflet and is modeled by $\mathrm{PC} / \mathrm{PS}, \mathrm{PC} / \mathrm{PS} / \mathrm{PIP} 2, \mathrm{PC} / \mathrm{PS} / \mathrm{PIP} 2 / \mathrm{K}$ or $\mathrm{PC} / \mathrm{PS} / \mathrm{PIP} 2 / \mathrm{Ca}$. The transmembrane potential difference is then calculated as $V_{m}=\psi(4)-$ $\psi(-4)$ and the results are shown in Fig. 2 b.
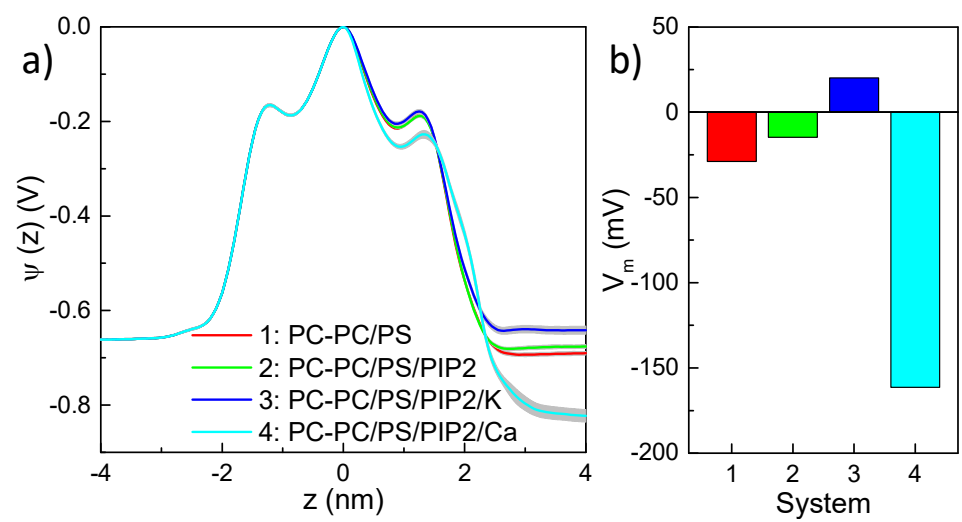

Figure 2. Effects of Membrane Asymmetry on Transmembrane Potential, $\psi(z)$, and transmembrane potential difference, $\boldsymbol{V}_{\boldsymbol{m}}$. (a) Electrostatic potential profile $\psi(z)$ across model membranes with asymmetric lipid composition (systems 1 and 2) and ion distribution (systems 2, 3 and 4); (b) The transmembrane potential difference $V_{m}$ in the four asymmetric membrane systems. 
PS and PS/PIP2 in the Inner Membrane Leaflet Contribute Differently to $V_{m}$. The electrostatic potential difference across the membranes $\left(V_{m}\right)$ was calculated as the difference in $\psi(z)$ values in the bulk water regions of the inner and outer leaflets: $V_{m}=\psi(4)-\psi(-4)$. For the reference symmetric pure POPC bilayer, the $\psi(z)$ values at $\mathrm{z}= \pm 4$ are identical at $-0.661 \mathrm{~V}$ (Fig. 1), yielding the expected $V_{m}=0 \mathrm{mV}$. Replacing $30 \%$ of the inner leaflet POPC lipids by POPS (net charge: $-1 e$ ) resulted in $\psi( \pm 4)=-0.690 \mathrm{~V}$ (Fig. 1), and thus decreasing the effective $V_{m}$ for the asymmetric PC-PC/PS system to $\sim-29 \mathrm{mV}$ (Fig. 2). This polarizing effect of POPS is consistent with previous united-atom MD simulation results ${ }^{[31]}$. Note that the change in $V_{m}$ is not caused by charge imbalance because counter ions were used for each of the PC and PC/PS simulations to keep the system neutral. Intriguingly, replacing a third of the POPS lipids with the polyvalent anionic lipid PIP2 (net charge: -5 e) changed $\psi( \pm 4)$ from $-0.690 \mathrm{~V}$ to $-0.676 \mathrm{~V}$ (Fig. 1 and Fig. 2a). In other words, the introduction of PIP2 decreases the $\left|V_{m}\right|$, where $V_{m}$ changes from $-29 \mathrm{mV}$ to $\sim-15 \mathrm{mV}$ (compare systems 1 and 2 in Fig. 2b). This shows that PIP2 counteracts the polarizing effect POPS, suggesting that the effect of anionic lipids on $V_{m}$ is not limited to their charge content. To check this further, we ran two additional simulations in which PIP2 was protonated at either the 4- or 5- position, thus reducing the effective charge by -1 e per PIP2 molecule. We obtained almost identical results for the protonated and regular PIP2 systems (Fig. S1), indicating that the effect of PS and PIP2 on the $V_{m}$ is probably a consequence of structure, dynamics, and specific interactions with counter ions rather than charge alone. This issue is discussed further in subsequent sections.

Asymmetric Ion Type Distribution Differently Affects $\boldsymbol{V}_{\boldsymbol{m}}$. In addition to lipid compositional asymmetry, the plasma membrane is also characterized by an asymmetric distribution of different ions. As shown Fig. 2, replacing $\mathrm{Na}^{+}$by $\mathrm{K}^{+}$in the PC/PS/PIP2 bilayer increased the electrostatic potential $\psi( \pm 4)$ from $-0.676 \mathrm{~V}$ to $-0.641 \mathrm{~V}$ (Fig. 1 and Fig. 2a), consistent with the effect of $K^{+}$ in resulting in a drop in the electrostatic potential across a pure dipalmitoylphosphatidylcholine (DPPC) bilayer observed in a previous simulation by Baker and colleagues ${ }^{[32]}$. As discussed below and in the reference ${ }^{[32]}$, this is due to the weaker $\mathrm{K}^{+}$-lipid interactions compared with $\mathrm{Na}^{+}$-lipid interactions. As a result of the reduced electrostatic potential, the effective $V_{m}$ for the asymmetric $\mathrm{PC}-\mathrm{PC} / \mathrm{PS} / \mathrm{PIP} 2$ bilayer changed from $-15 \mathrm{mV}$ in the presence of sodium to $+20 \mathrm{mV}$ when sodium is replaced by potassium (compare to systems 2 and 3 in Fig. 2b). A similar observation has been made in a previous simulation of a POPC/1-palmitoyl-2-oleoyl-sn-glycero-3phosphoethanolamine (POPE) bilayer ${ }^{[33]}$. In this study, $K^{+}$was found to neutralize the intrinsic transmembrane potential in an asymmetric POPC (outer)/POPE (inner) bilayer. Together, these observations suggest that the electrostatic interaction of sodium and potassium ions with lipids, and hence their local distribution in and near the bilayer, is completely different despite their identical charge. Therefore, to the extent that the CHARMM36 force field used here faithfully captures the solvation properties and detailed interactions of these ions with each of the PC, PS and PIP2 lipids studied in this work, we conclude that the interplay among the various ion-lipid interactions -rather than just the charge -- is critical for the magnitude and direction of the transmembrane potential. 
In order to further probe the effect of ion type asymmetric distribution on $V_{m}$, we considered the divalent ion $\mathrm{Ca}^{2+}$, simulated at the non-physiological concentration of $\sim 75 \mathrm{mM}$ so that the overall charge content remains the same as in the $\mathrm{Na}^{+}$and $\mathrm{K}^{+}$systems. As shown in Fig. 2, relative to $\mathrm{Na}^{+}, \mathrm{Ca}^{2+}$ decreased the $\psi( \pm 4)$ of the PC/PS/PIP2 bilayer from $-0.676 \mathrm{~V}$ to $-0.823 \mathrm{~V}$ (Fig. 1 and Fig. 2a). As a result, the effective $V_{m}$ in the asymmetric PC-PC/PS/PIP2 membrane changed from $-15 \mathrm{mV}$ to $-162 \mathrm{mV}$ (systems 2 and 4 in Fig. 2b). As shown below, this large effect of calcium in polarizing the three-component model membrane is directly correlated with its strong interactions with lipid head groups, confirming that the detailed ion-lipid interactions are responsible for the differential effect of ions on $V_{m}$. Overall, our simulations clearly show that, for the threecomponent PC/PS/PIP2 bilayer, the asymmetric distribution of different cations affects the $V_{m}$ differently--either depolarizing $\left(\mathrm{K}^{+}\right)$or polarizing $\left(\mathrm{Ca}^{2+}\right)$ the membrane relative to $\mathrm{Na}^{+}-{ }_{-}$ depending on their specific interactions with the constituent lipids.

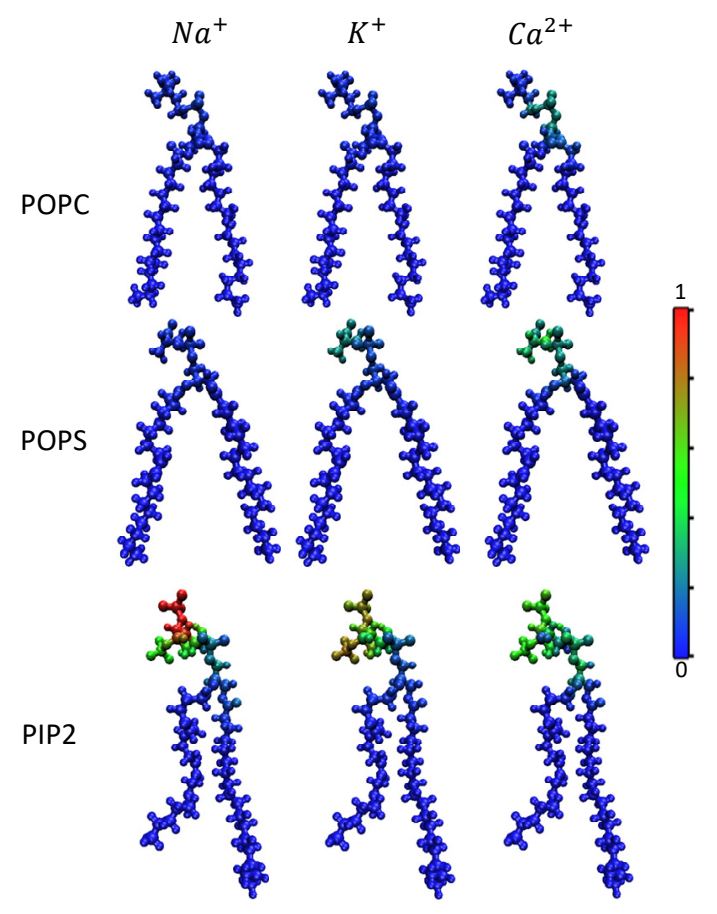

Figure 3. Contact probability between ions and lipids. Atoms in lipids were colored blue through red based on their contact probability with ions.

\section{Differential Interactions of $\mathrm{Na}^{+}, \mathrm{K}^{+}$and $\mathrm{Ca}^{2+}$ with Lipids Underlies their Differential Effect} on $\boldsymbol{V}_{\boldsymbol{m}}$. To examine how and the extent to which differential cation-lipid interactions modulate the magnitude and direction of $V_{m}$, we quantified the time- and ensemble-averaged contact probability $\mathrm{Na}^{+}, \mathrm{K}^{+}$and $\mathrm{Ca}^{2+}$ with each atom of each lipid type (Fig. 3). As expected, electrostatic interactions dominate the ion-lipid contacts, so that the PIP2 head group (net charge: $-5 e$ ) exhibits the largest contact probability. Compared to the POPC pure bilayer, the presence of the anionic lipids strengthened the adsorption of the three cations onto the membrane ${ }^{[34]}$. In fact, except for $\mathrm{Ca}^{2+}$ which interacts with the phosphate group of each lipid type with comparable probability, 
$\mathrm{Na}^{+}$and $\mathrm{K}^{+}$have no measureable interactions with POPC. This, however, does not rule out lowresident contacts as these cations are able to dynamically interact with the negatively charged phosphate group. The unexpectedly substantial interaction of $\mathrm{Ca}^{2+}$ with the phosphate groups of PC, PS and PIP2 lipids, however, is likely due to its excessive attraction to phosphates in the CHARMM36 force field, as observed previously ${ }^{[35]}$.

The three cations also differ in their capacity to interact with the PS and PIP2 lipids. Intriguingly, $K^{+}$exhibits a higher tendency of interacting with the POPS carboxyl (and to a lesser extent phosphate) oxygen atoms than $\mathrm{Na}^{+}$(Fig. 3); in contrast, $\mathrm{Na}^{+}$interacts much more strongly with the sugar phosphates of PIP2. These differential affinities of $\mathrm{Na}^{+}$and $\mathrm{K}^{+}$for PS and PIP2 lipids, coupled with the slightly larger aggregate number of $\mathrm{Na}^{+}$sequestered by PIP2, likely explains the opposite effect of these cations on the direction of the $V_{m}$ of the PC-PC/PS/PIP2 system (Fig. 2). $\mathrm{Ca}^{2+}$, on the other hand, extensively interacts with both the carboxyl oxygen atoms of POPS and the sugar phosphates of PIP2, in addition to its interaction with the ester phosphate of all three lipid types. Taken together, the differential interactions of the three cations with the anionic moieties of the three lipid types explains their dramatically different effects on the direction and magnitude of the effective transmembrane potential of the asymmetric systems studied in this work.

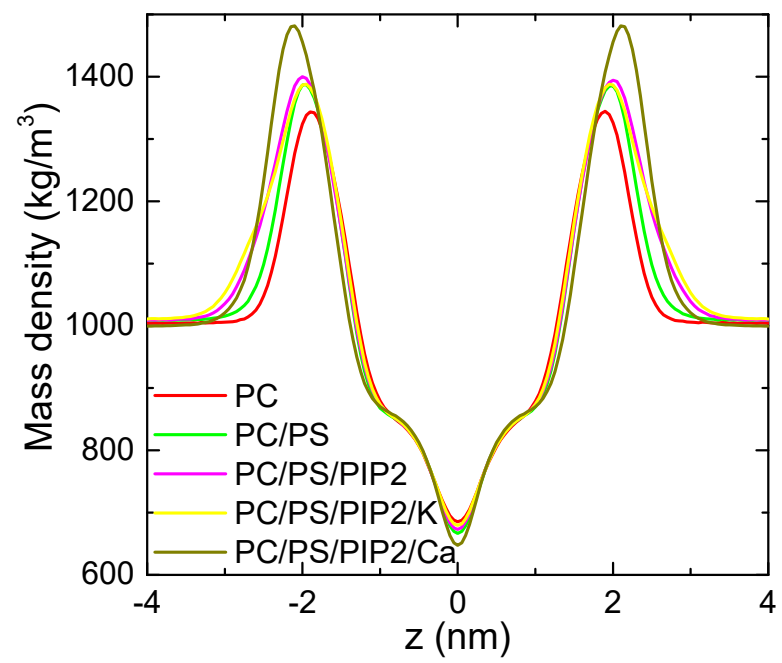

Figure 4. Mass density distribution profile of the model membrane systems studied in this work.

Structural Details of the Membrane Systems Simulated in This Work. The extent of bilayer penetration by ions can be modulated by the local area per lipid ${ }^{[36]}$, and vice versa. In other words, changes in membrane composition and the resulting change in membrane tension could alter lipidion interactions, which in turn affects the transmembrane potential. To quantitatively investigate this issue, we calculated the mass density distribution and the acyl chain order of each simulated system. As shown in Fig. 4, the addition of POPS or POPS/PIP2 in the PC bilayer reshaped the mass density distribution along the membrane normal. For example, relative to the pure PC system, the membrane thickness (peak-to-peak distance in Fig. 4) increased by approximately the same magnitude in both PC/PS and PC/PS/PIP2. Furthermore, $\mathrm{Na}^{+}$increased the thickness of the PC/PS/PIP2 bilayer slightly more than $\mathrm{K}^{+}$(compare purple and yellow in Fig. 4), whereas $\mathrm{Ca}^{2+}$ 
does so even more strongly (dark yellow). Similarly, the width and height of the mass density distributions indicate differential adsorption of the ions across the head group region. These effects are directly correlated with the differential impact of the cations on the $V_{m}$ (Fig. 2). Note that the difference in peak height and width of the distributions among PC, PC/PS and PC/PS/PIP2 bilayers in the presence of $\mathrm{Na}^{+}$(i.e. red, green and purple in Fig. 4) is largely due to the larger size of the head group in PS and PIP2 relative to PC (see Fig. 6a).
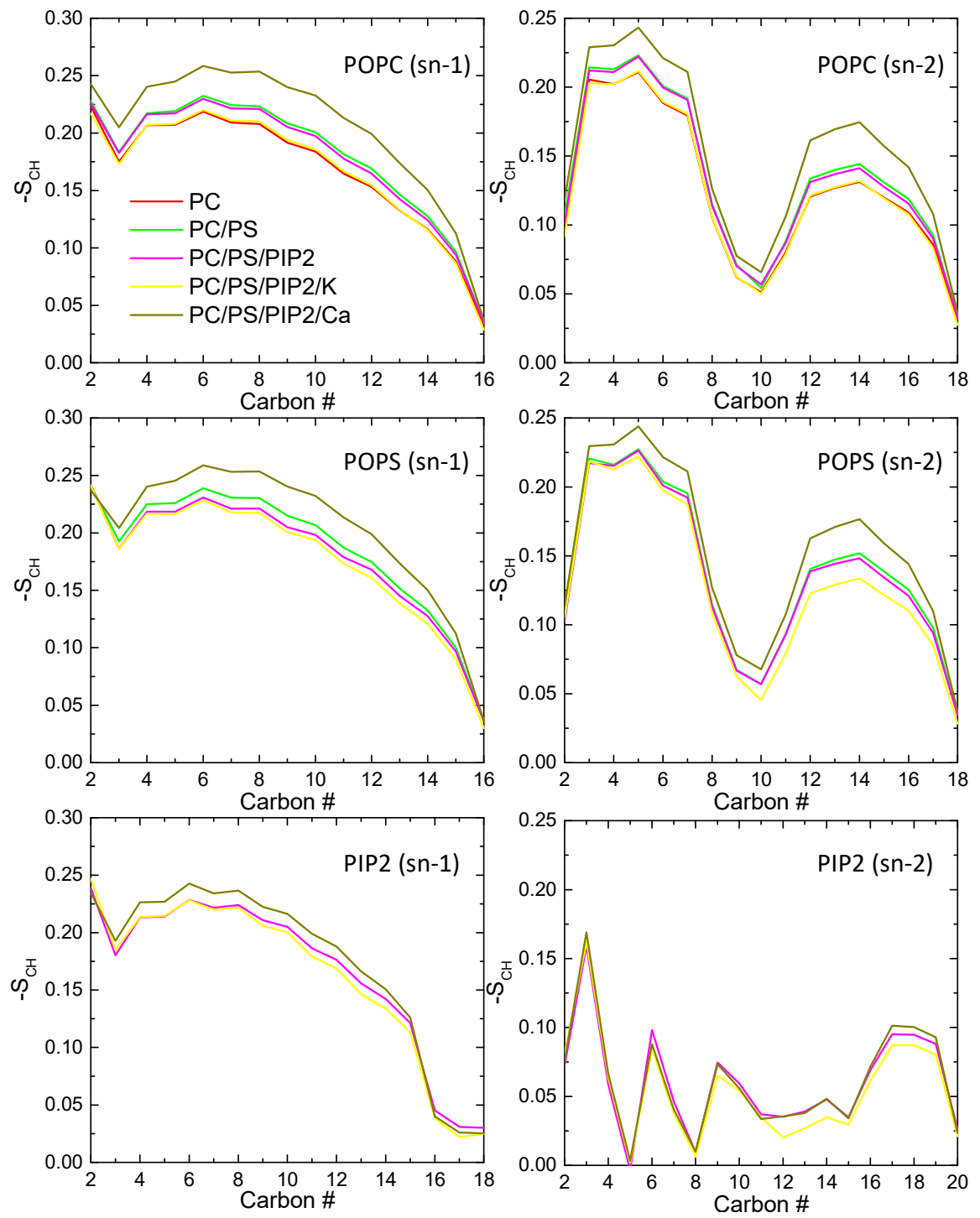

Figure 5. Lipid chain order parameter of the five simulated symmetric membranes in this work.

Since membrane thickness is correlated with lipid chain order, we calculated the NMR order parameter for each chain (sn-1 and sn-2) of each lipid type in the five symmetric membrane systems simulated in this work (Table 1). Fig. 5 (top) shows that, the presence of 30\% POPS made both chains of the POPC lipids more ordered (compare system PC (red) and PC/PS (green)). This, 
however, could also be due to the presence of more $\mathrm{Na}^{+}$ions in the PC/PS bilayer. When one-third of the PS lipids in the PC/PS bilayer is replaced by PIP2, no significant changes occurred to the POPC lipid chain order (Fig. 5, top), although the sn-1 order parameter of the PS lipids is reduced slightly (Fig 5, middle). The latter is likely due to the even larger number of $\mathrm{Na}^{+}$ions in the PC/PS/PIP2 bilayer (see Table 1). The impact of the cations on lipid chain order is further demonstrated by the fact that the three cations have different ordering effects. First, compared to $\mathrm{Na}^{+}, \mathrm{K}^{+}$rendered both the sn-2 chains of both POPC and POPS more disordered, despite the identical number and valence of the two ions in the system (purple and yellow in Fig. 5). This may be partly explained by the slightly higher affinity of $K^{+}$for the PS lipids (Fig. 3). Unlike the ordering effect of $\mathrm{K}^{+}, \mathrm{Ca}^{2+}$ induced a dramatic increase in the order of both lipid chains of all three lipid types. Each of these observations are directly concordant with the effect of the cations on the $V_{m}$ (Fig. 2). We conclude that lipid composition and especially the number and type of cations affect lipid packing and thus the charge density distribution along the membrane normal, which inevitably modulates the electrostatic potential of the model membranes and thereby the transmembrane potential $V_{m}$.

Impact of Cation-Lipid Interactions on Lipid Head Group Orientation. As mentioned above, $\mathrm{K}^{+}$and $\mathrm{Ca}^{2+}$ ions have a notable effect on lipid chain order. To further examine the effect of these ions on membrane structure, we characterized the orientation of lipid head groups in each of the three-component bilayers systems PC/PS/PIP2, PC/PS/PIP2/K, PC/PS/PIP2/Ca. We defined lipid head group orientation by the tilt angle from the membrane normal of a vector along the $\mathrm{P}-\mathrm{N}$ bond (for PC and PS) and across the six-membered rings (for PIP2), as shown in Fig. 6a. A smaller tilt angle indicates a better alignment of the lipid head group along the membrane normal, which we refer to as "ordered lipid head orientation". Conversely, when the tilt angle is larger, the head group is considered to be orientationally "disordered". We found that, when compared to $\mathrm{Na}^{+}, \mathrm{K}^{+}$ induced a slight disorder in the POPC lipids and order to the POPS lipids, respectively, and a more dramatic orientational order to the PIP2 lipid head groups (Fig. 6b). In contrast, $\mathrm{Ca}^{2+}$ significantly ordered the orientation of POPC lipids and disordered the POPS and PIP2 lipids (Fig. 6b).

In addition to being correlated with the trend of the $V_{m}$ (Fig. $\mathbf{2 b}$ ), the effect of the three cations on head group orientation is broadly consistent with the strength of their interaction with anionic moieties in the different lipids (see Fig. 3). For example, when ranked by contact probability with the ester phosphate of POPC, the carboxyl group of POPS and the sugar phosphate of PIP2, we obtain $\mathrm{Na}^{+} \approx \mathrm{K}^{+}<\mathrm{Ca}^{2+}, \mathrm{Na}^{+}<\mathrm{K}^{+}<\mathrm{Ca}^{2+}$ and $\mathrm{Na}^{+}>\mathrm{K}^{+}>\mathrm{Ca}^{2+}$, respectively. When ranked by lipid head group orientational order, the trend is $\mathrm{Na}^{+}>\mathrm{K}^{+}<\mathrm{Ca}^{2+}, \mathrm{Na}^{+} \approx \mathrm{K}^{+}>\mathrm{Ca}^{2+}$ and $\mathrm{Na}^{+}<\mathrm{K}^{+}>\mathrm{Ca}^{2+}$, respectively (Fig. 6b). The imperfect correlation suggests that the differential effect of the ions on lipid head orientation may also be affected by non-specific ion-lipid interactions. This can be captured qualitatively by examining the extent to which the cations are adsorbed on the membrane surface. As shown Fig. 6c, there are substantial differences in the generalized adsorption of the different ions on the membrane. In particular, almost all $\mathrm{Ca}^{2+}$ ions have moved from the bulk solution to embed in the lipid head region. In contrast, $\mathrm{Na}^{+}$and $\mathrm{K}^{+}$ ions, which have relatively weaker interaction with lipid head groups, tend to dynamically exchange with ions in the bulk water. Taken together and assuming negligible force field artifacts, these results suggest that induction of head group orientational disorder in the zwitterionic POPC 
and order in the anionic POPS and PIP2 lipids by a cation such as $K^{+}$depolarizes an asymmetric PC-PC/PS/PIP2 bilayer. Conversely, cations such $\mathrm{Ca}^{2+}$ that disorder the head group of POPS and PIP2 and order that of POPC polarize the bilayer.

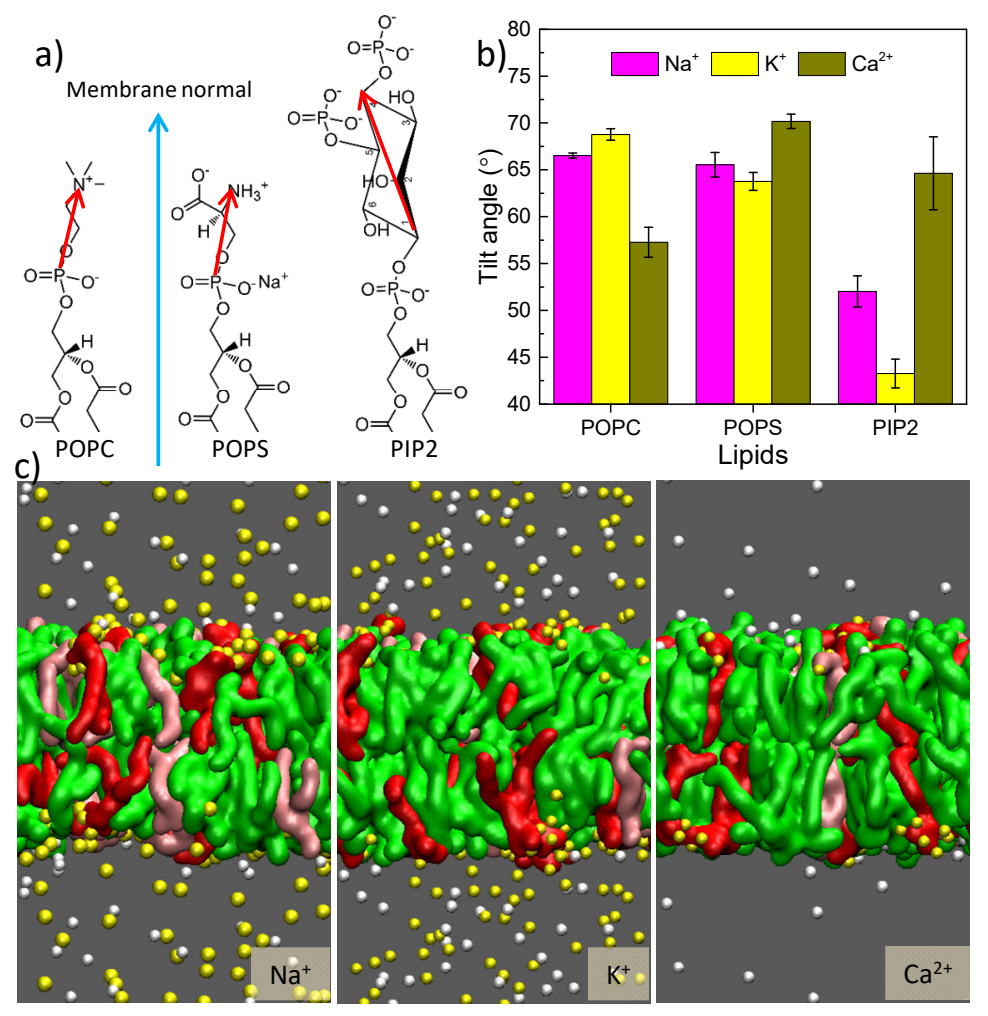

Figure 6. Effects of cations on lipid head-group orientation. (a) Schematics of the vectors of lipid head groups used for calculating tilt angle. (b) Average tilt angles of the three lipids in the presence of different cations. Errors represent the standard deviation of the average over the last three 300ns trajectories. (c) Snapshots of the three systems with different cations. POPC is in green, POPS in pink, PIP2 in red. $\mathrm{Na}^{+}, \mathrm{K}^{+}$and $\mathrm{Ca}^{2+}$ are in yellow, and $\mathrm{Cl}^{-}$in white. Water molecules are omitted for clarity.

Overall, our simulations suggest that, at least for POPS and PIP2 lipids, the impact of anionic charge on the transmembrane potential difference could be dramatically different and depends not only charge content but also structure and dynamics. Different lipid species and ion types can bring about different ion-lipid and lipid-lipid interactions that jointly regulate membrane equilibrium structure and charge distribution along the membrane normal. Hence, steady state or transient asymmetric alterations in either lipid composition or ion distribution in the intracellular and extracellular space of the cell may cause major changes in the transmembrane potential difference $V_{m}$.

\section{Conclusion}


In this work, symmetric model membrane systems were used in all-atom MD simulations to evaluate the electrostatic potential profiles of physiologically relevant asymmetric membranes. Averaging of the two halves of symmetric membrane systems can ensure the symmetric charge density distribution around the membrane center, which yields a reliable $\psi(z)$ profile and thus eliminates a non-zero $V_{m}$ in multi-component symmetric model membranes. We have shown that one could obtain a recombinant $\psi(z)$ profile for an asymmetric membrane by combining two symmetrized $\psi(z)$ profiles obtained from two independent MD simulations, one modeling the inner leaflet and another modeling the outer leaflet of the plasma membrane. In the current work, a pure POPC bilayer in a $150 \mathrm{mM} \mathrm{NaCl}$ solution was used as a model for the outer half of the plasma membrane. The inner leaflet was modeled by a two-component POPC/POPS and a threecomponent POPC/POPS/PIP2 bilayer in the presence of neutralizing $\mathrm{Na}^{+}$ions. The POPC/POPS/PIP2 bilayer was also simulated after replacing $\mathrm{Na}^{+}$with $\mathrm{K}^{+}$or $\mathrm{Ca}^{2+}$ ions. Comparison of the effective transmembrane potential difference $V_{m}$ obtained from combining the $\psi(z)$ profiles of the POPC bilayer with each of the inner leaflet model membranes indicated inner leaflet POPS in the inner membrane leaflet polarizes the membrane $\left(\Delta V_{m}<0\right)$ whereas replacing a third of the PS lipids by PIP2 partially counteracts this effect. Furthermore, replacing intracellular $\mathrm{Na}^{+}$by $\mathrm{K}^{+}$depolarized the membrane $\left(\Delta V_{m}>0\right)$ while $\mathrm{Ca}^{2+}$ further polarizes the membrane. We have shown that these differential effects are a result of differential cation-lipid interactions, and are correlated with changes in lipid chain order and head group orientation.

\section{Supporting Information}

Additional figures of charge density distribution and electrostatic potential profiles are provided.

\section{Acknowledgements}

This work was supported by the National Natural Science Foundation of China (No. 21903002), the Fundamental Research Funds for the Central Universities (No. YWF-20-BJ-J-632), Open Fund of State Key Laboratory of Membrane Biology (No. 2020KF09) to XL, and NIH funding R01GM124233 to AAG. We are grateful to the Texas Advanced Computing Center (TACC), the Extreme Science and Engineering Discovery Environment (XSEDE) and the Supercomputing Center of Beihang University for generous computing resources.

\section{Data Availability Statement}

The data that support the findings of this study are available from the corresponding authors upon the reasonable request.

\section{References:}


[1] Alberts, B.; Johnson, A.; Lewis, J.; Morgan, D.; Raff, M.; Roberts, K.; Walter, P., Molecular Biology of the Cell (6th Edition). Garland Press: New York, 2014.

[2] Shu, Y.; Hasenstaub, A.; Duque, A.; Yu, Y.; McCormick, D. A., Modulation of Intracortical Synaptic Potentials by Presynaptic Somatic Membrane Potential. Nature, 2006, 441, 761-765.

[3] Gao, X.; Hong, S.; Liu, Z.; Yue, T.; Dobnikar, J.; Zhang, X., Membrane potential drives direct translocation of cell-penetrating peptides. Nanoscale, 2019, 11, 1949-1958.

[4] Thrivikraman, G.; Boda, S. K.; Basu, B., Unraveling the Mechanistic Effects of Electric Field Stimulation towards Directing Stem Cell Fate and Function: A Tissue Engineering Perspective. Biomaterials, 2017.

[5] Yang, M.; Brackenbury, W. J., Membrane Potential and Cancer Progression. Front. Physiol., 2013, 4.

[6] Zhou, Y.; Wong, C.-O.; Cho, K.-j.; van der Hoeven, D.; Liang, H.; Thakur, D. P.; Luo, J.; Babic, M.; Zinsmaier, K. E.; Zhu, M. X.; Hu, H.; Venkatachalam, K.; Hancock, J. F., Membrane Potential Modulates Plasma Membrane Phospholipid Dynamics and K-Ras Signaling. Science, 2015, 349, 873 876.

[7] Van Meer, G.; Voelker, D. R.; Feigenson, G. W., Membrane Lipids: Where They Are and How They Behave. Nat. Rev. Mol. Cell Biol., 2008, 9, 112.

[8] Levental, K. R.; Lorent, J. H.; Lin, X.; Skinkle, A. D.; Surma, M. A.; Stockenbojer, E. A.; Gorfe, A. A.; Levental, I., Polyunsaturated Lipids Regulate Membrane Domain Stability by Tuning Membrane Order. Biophys. J., 2016, 110, 1800-1810.

[9] Bilkova, E.; Pleskot, R.; Rissanen, S.; Sun, S.; Czogalla, A.; Cwiklik, L.; Róg, T.; Vattulainen, I.; Cremer, P. S.; Jungwirth, P., Calcium Directly Regulates Phosphatidylinositol 4, 5-bisphosphate Headgroup Conformation and Recognition. J. Am. Chem. Soc, 2017, 139, 4019-4024.

[10] Wen, Y.; Vogt, V. M.; Feigenson, G. W., Multivalent Cation-Bridged PI(4,5)P2 Clusters Form at Very Low Concentrations. Biophys. J., 2018, 114, 2630-2639.

[11] Di Paolo, G.; De Camilli, P., Phosphoinositides in Cell Regulation and Membrane Dynamics. Nature, 2006, 443, 651-657.

[12] Shi, X.; Bi, Y.; Yang, W.; Guo, X.; Jiang, Y.; Wan, C.; Li, L.; Bai, Y.; Guo, J.; Wang, Y.; Chen, X.; Wu, B.; Sun, H.; Liu, W.; Wang, J.; Xu, C., Ca2+ Regulates T-cell Receptor Activation by Modulating the Charge Property of Lipids. Nature, 2013, 493, 111-115.

[13] Prakash, P.; Zhou, Y.; Liang, H.; Hancock, J. F.; Gorfe, A. A., Oncogenic K-Ras Binds to an Anionic Membrane in Two Distinct Orientations: A Molecular Dynamics Analysis. Biophys. J., 2016, 110, 1125-1138.

[14] Delemotte, L.; Dehez, F.; Treptow, W.; Tarek, M., Modeling Membranes under a Transmembrane Potential. J. Phys. Chem. B, 2008, 112, 5547-5550.

[15] Melcr, J.; Bonhenry, D.; Timr, S. t. p. n.; Jungwirth, P., Transmembrane Potential Modeling: Comparison between Methods of Constant Electric Field and Ion Imbalance. J. Chem. Theory Comput., 2016, 12, 2418-2425.

[16] Basdevant, N.; Dessaux, D.; Ramirez, R., Ionic Transport through a Protein Nanopore: a CoarseGrained Molecular Dynamics Study. Sci. Rep., 2019, 9, 15740.

[17] Böckmann, R. A.; Hac, A.; Heimburg, T.; Grubmüller, H., Effect of Sodium Chloride on a Lipid Bilayer. Biophys. J., 2003, 85, 1647-1655.

[18] Lin, X.; Nair, V.; Zhou, Y.; Gorfe, A. A., Membrane Potential and Dynamics in a Ternary Lipid Mixture: Insights from Molecular Dynamics Simulations. Phys. Chem. Chem. Phys., 2018, 20, 1584115851.

[19] Li, H.; Chowdhary, J.; Huang, L.; He, X.; MacKerell, A. D.; Roux, B., Drude Polarizable Force Field for Molecular Dynamics Simulations of Saturated and Unsaturated Zwitterionic Lipids. J. Chem. Theory Comput., 2017, 13, 4535-4552.

[20] Huang, J.; Rauscher, S.; Nawrocki, G.; Ran, T.; Feig, M.; de Groot, B. L.; Grubmüller, H.; MacKerell, A. D., CHARMM36m: An Improved Force Field for Folded and Intrinsically Disordered Proteins. Nat. Methods, 2017, 14, 71-73. 
[21] Falkovich, S. G.; Martinez-Seara, H.; Nesterenko, A. M.; Vattulainen, I.; Gurtovenko, A. A., What Can We Learn about Cholesterol's Transmembrane Distribution Based on Cholesterol-Induced Changes in Membrane Dipole Potential? J. Phys. Chem. Lett., 2016, 7, 4585-4590.

[22] Jo, S.; Kim, T.; Iyer, V. G.; Im, W., CHARMM-GUI: A Web-based Graphical User Interface for CHARMM. J. Comput. Chem., 2008, 29, 1859-1865.

[23] Lee, J.; Cheng, X.; Swails, J. M.; Yeom, M. S.; Eastman, P. K.; Lemkul, J. A.; Wei, S.; Buckner, J.; Jeong, J. C.; Qi, Y.; Jo, S.; Pande, V. S.; Case, D. A.; Brooks, C. L.; MacKerell, A. D.; Klauda, J. B.; Im, W., CHARMM-GUI Input Generator for NAMD, GROMACS, AMBER, OpenMM, and CHARMM/OpenMM Simulations Using the CHARMM36 Additive Force Field. J. Chem. Theory Comput., 2016, 12, 405-413.

[24] Nosé, S., A Molecular Dynamics Method for Simulations in the Canonical Ensemble. Mol. Phys., 1984, 52, 255-268.

[25] Hoover, W. G., Canonical Dynamics: Equilibrium Phase-Space Distributions. Phys. Rev. A, 1985, 31, 1695-1697.

[26] Parrinello, M.; Rahman, A., Polymorphic Transitions in Single Crystals: A New Molecular Dynamics Method. J. Appl. Phys., 1981, 52, 7182-7190.

[27] Hess, B.; Bekker, H.; Berendsen, H. J. C.; Fraaije, J. G. E. M., LINCS: A Linear Constraint Solver for Molecular Simulations. J. Comput. Chem., 1997, 18, 1463-1472.

[28] Essmann, U.; Perera, L.; Berkowitz, M. L.; Darden, T.; Lee, H.; Pedersen, L. G., A Smooth Particle Mesh Ewald Method. J. Chem. Phys., 1995, 103, 8577-8593.

[29] Abraham, M. J.; Murtola, T.; Schulz, R.; Páll, S.; Smith, J. C.; Hess, B.; Lindahl, E., GROMACS: High Performance Molecular Simulations through Multi-Level Parallelism from Laptops to Supercomputers. SoftwareX, 2015, 1-2, 19-25.

[30] Humphrey, W.; Dalke, A.; Schulten, K., VMD: Visual Molecular Dynamics. J. Mol. Graph., 1996, 14, 33-38.

[31] Gurtovenko, A. A.; Vattulainen, I., Membrane Potential and Electrostatics of Phospholipid Bilayers with Asymmetric Transmembrane Distribution of Anionic Lipids. J. Phys. Chem. B, 2008, 112, 46294634.

[32] Lee, S.-J.; Song, Y.; Baker, N. A., Molecular Dynamics Simulations of Asymmetric $\mathrm{NaCl}$ and $\mathrm{KCl}$ Solutions Separated by Phosphatidylcholine Bilayers: Potential Drops and Structural Changes Induced by Strong Na+-Lipid Interactions and Finite Size Effects. Biophys. J., 2008, 94, 3565-3576.

[33] Gurtovenko, A. A.; Vattulainen, I., Intrinsic Potential of Cell Membranes: Opposite Effects of Lipid Transmembrane Asymmetry and Asymmetric Salt Ion Distribution. J. Phys. Chem. B, 2009, 113, 71947198.

[34] Jurkiewicz, P.; Cwiklik, L.; Vojtǐšková, A.; Jungwirth, P.; Hof, M., Structure, Dynamics, and Hydration of POPC/POPS Bilayers Suspended in $\mathrm{NaCl}, \mathrm{KCl}$, and $\mathrm{CsCl}$ Solutions. Biochim. Biophys. Acta-Biomembr., 2012, 1818, 609-616.

[35] Han, K.; Venable, R. M.; Bryant, A.-M.; Legacy, C. J.; Shen, R.; Li, H.; Roux, B.; Gericke, A.; Pastor, R. W., Graph-Theoretic Analysis of Monomethyl Phosphate Clustering in Ionic Solutions. J. Phys. Chem. B, 2018, 122, 1484-1494.

[36] Javanainen, M.; Melcrová, A.; Magarkar, A.; Jurkiewicz, P.; Hof, M.; Jungwirth, P.; Martinez-Seara, H., Two Cations, Two Mechanisms: Interactions of Sodium and Calcium with Zwitterionic Lipid Membranes. Chem. Commun., 2017, 53, 5380-5383. 


\title{
Supporting Information for
}

\section{Transmembrane Potential of Physiologically Relevant Model Membranes: Effects of Membrane Asymmetry}

\author{
Xubo Lin ${ }^{1, *}$, Alemayehu A. Gorfe ${ }^{2, *}$
}

1. Institute of Single Cell Engineering, Beijing Advanced Innovation Center for Biomedical Engineering, Beihang University, Beijing 100191, China

2. Department of Integrative Biology and Pharmacology, McGovern Medical School, The University of Texas Health Science Center at Houston, Texas 77030, USA

Correspondence to: linxbseu@buaa.edu.cn (XL) and alemayehu.g.abebe@uth.tmc.edu (AAG)

The authors declare no competing financial interest.
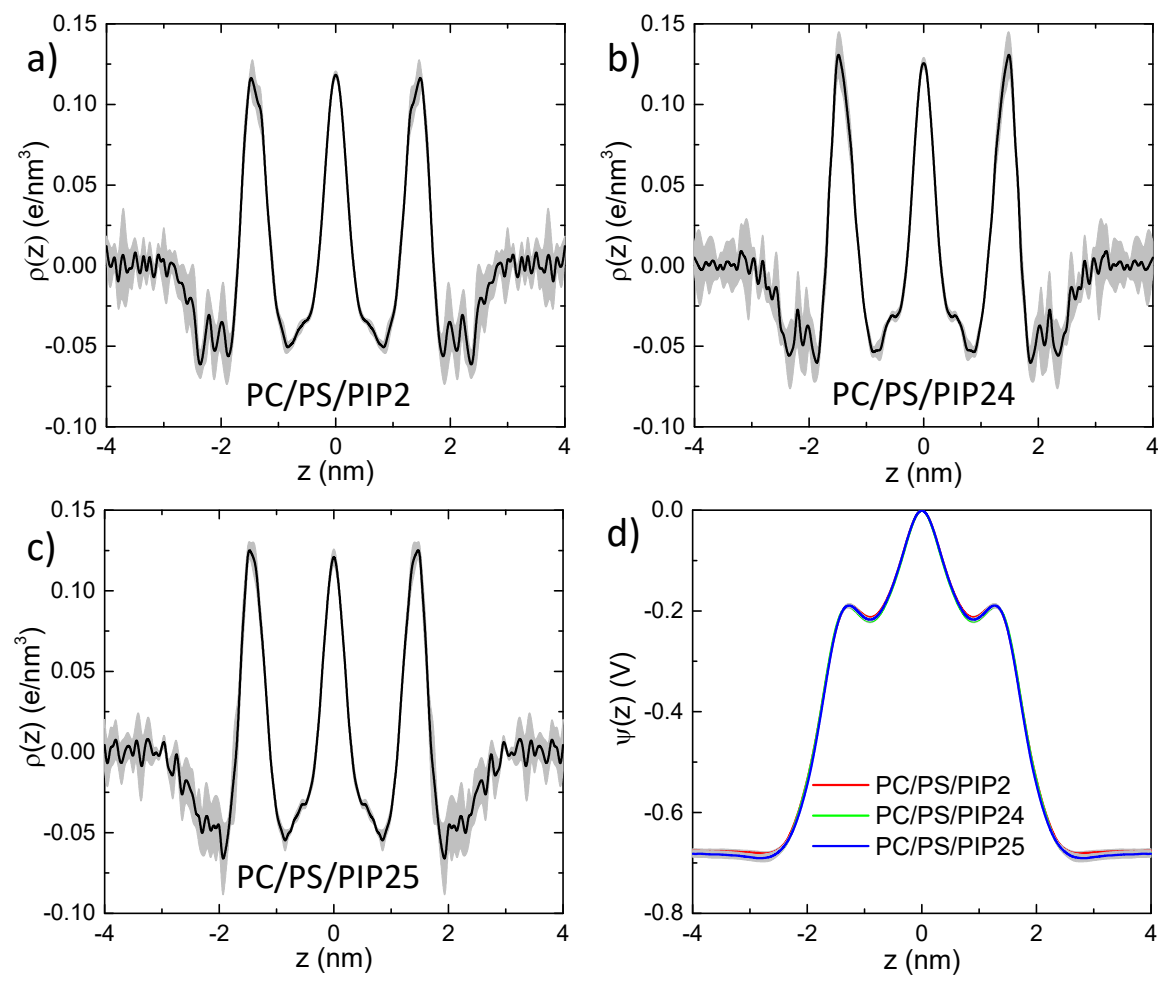

Figure S1. Charge density distribution profiles for system PC/PS/PIP2 (a), PC/PS/PIP24 (b) and PC/PS/PIP25 (c). as well as their electrostatic potential profiles (d). PIP24 (net charge: $-4 e$ ) and PIP25 (net charge: $-4 e$ ) are 
the PIP2 lipids with one protonation at 4- and 5- positions respectively. The results clearly indicated that the PIP2 protonation had no significant effects of the electrostatic potential distributions of model membranes. 